\title{
DBQ: A Differentiable Branch Quantizer for Lightweight Deep Neural Networks
}

\author{
Hassan Dbouk $^{1,2 \star}$, Hetul Sanghvi ${ }^{2}$, Mahesh Mehendale ${ }^{2}$, and Naresh \\ Shanbhag ${ }^{1}$ \\ 1 Dept. of Electrical and Computer Engineering, University of Illinois at \\ Urbana-Champaign, Urbana, USA \\ \{hdbouk2, shanbhag\}@illinois.edu \\ 2 Kilby Labs, Texas Instruments Inc, Dallas, USA \\ \{hetul, m-mehendale\}@ti.com
}

\begin{abstract}
Deep neural networks have achieved state-of-the art performance on various computer vision tasks. However, their deployment on resource-constrained devices has been hindered due to their high computational and storage complexity. While various complexity reduction techniques, such as lightweight network architecture design and parameter quantization, have been successful in reducing the cost of implementing these networks, these methods have often been considered orthogonal. In reality, existing quantization techniques fail to replicate their success on lightweight architectures such as MobileNet. To this end, we present a novel fully differentiable non-uniform quantizer that can be seamlessly mapped onto efficient ternary-based dot product engines. We conduct comprehensive experiments on CIFAR-10, ImageNet, and Visual Wake Words datasets. The proposed quantizer (DBQ) successfully tackles the daunting task of aggressively quantizing lightweight networks such as MobileNetV1, MobileNetV2, and ShuffleNetV2. DBQ achieves state-ofthe art results with minimal training overhead and provides the best (pareto-optimal) accuracy-complexity trade-off.
\end{abstract}

Keywords: Deep Learning, Quantization, Low-Complexity Neural Networks

\section{Introduction}

Deep neural networks (DNNs) have achieved state-of-the art accuracy on various computer vision tasks such as image classification $[15,6]$ but at the expense of extremely high computational and storage complexity, e.g., ResNet-18 [6] needs $\sim 10^{12}$ 1-b full adders (FAs) and $3.74 \times 10^{8}$-bits of activation and weight storage to achieve an accuracy of $70 \%$ on the ImageNet dataset. These high computational and storage costs inhibit the deployment of such DNNs on resource-constrained Edge devices. As a result, there is much interest in designing low-complexity DNNs without compromising their accuracy.

\footnotetext{
* Work done while at Kilby Labs.
} 


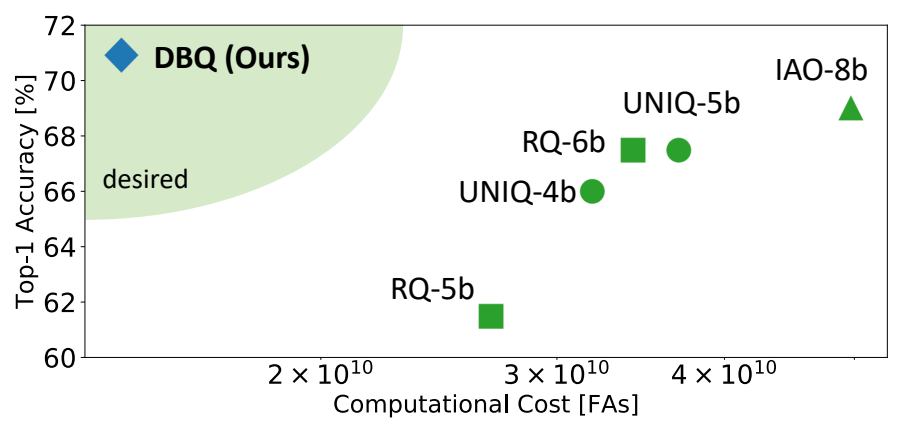

Fig. 1. The Top-1 accuracy on ImageNet vs. computational cost for MobileNetV1 achieved by state-of-the-art quantization methods (RQ [19], UNIQ [1], and IAO [13]). Our proposed method DBQ simultaneously achieves the highest accuracy and the lowest complexity.

There are two distinct approaches for reducing DNN complexity: 1) model compression [5] and quantization [10,22] of complex networks, and 2) the design of lightweight networks from scratch, e.g., MobileNet [8,27].

Model compression and quantization methods rely on the intrinsic over parameterization in complex networks to reduce their complexity. Such methods have proved to be very effective in reducing network complexity with negligible impact on its accuracy, e.g., ternary quantization of ResNet-18 weights [32] reduces its computational and storage complexity by $88 \%$ and $74 \%$, respectively, at the expense of a drop in accuracy from $70.3 \%$ to $69.1 \%$.

In the second approach, the design of lightweight networks such as MobileNet [8,27], SqueezeNet [11], ShuffleNet [34], ConDenseNet [9] have also shown tremendous success. Such networks exploit algorithmic properties such as factorizability of convolutions and utilize either $1 \times 1$ convolutions (SqueezeNet), grouped convolutions (ShuffleNet, ConDenseNet), or both (MobileNet). For example, MobileNetV1 [8] achieves comparable (or even higher) accuracy than its ResNet-18 floating-point (FP) counterpart but at a computational and storage complexity that are $3 \times$ and $7 \times$ lower, respectively.

In contrast, not much work has been done in model compression or quantization of lightweight networks and for a good reason - such networks are already irredundant leaving much less room for complexity reduction. Existing works $[19,30,1,13,28]$ that quantize lightweight networks use fixed-point quantization with relatively high bitwidths (see Fig. 1) which offer limited reductions in complexity. In contrast, aggressive quantization schemes such as binarization $[10,22]$ or ternarization $[36,16]$ have been benchmarked on over-parameterized networks. In fact, ternarizing MobileNetV1 leads to a catastrophic drop in accuracy from $72.12 \%$ to $66.45 \%$ on ImageNet as we show in Section 4.3. In order to improve the performance of ternarized models while leveraging the simplicity of ternary-based arithmetic, one can construct a non-uniform quantizer as linear combinations of ternary values. Such formulation has already been proposed in 
the context of binarized neural networks [33,18], however the training algorithm involved is: 1) extremely inefficient to implement; 2) can lead to sub-optimal results due to gradient mismatch issues; and 3) has only been benchmarked on over-parameterized networks.

To this end, our work is the first to tackle the daunting task of aggressively quantizing lightweight networks, such as MobileNetV1 [8], MobileNetV2 [27], and ShuffleNetV2 [20] using multiple ternary branches. We propose an efficient and fully differentiable multiple ternary branch quantization algorithm (DBQ). For MobileNetV1 on ImageNet, DBQ achieves an accuracy $2 \%$ higher than stateof-the art quantization methods with a complexity that is $3.5 \times$ lower as shown in Fig. 1. This represents an overall reduction of $24.5 \times$ compared to FP with a $1.2 \%$ drop in accuracy.

Specifically, our contributions are:

1. We are the first to successfully ternarize lightweight networks (MobileNetV1, MobileNetV2, ShuffleNetV2) on ImageNet. This result is achieved by using DBQ with two ternary branches.

2. We present the first fully differentiable branched quantization algorithm (DBQ) for DNNs requiring minimal training overhead.

3. We show that DBQ outperforms state-of-the art methods in both accuracy and computational cost. Compared to state-of-the art quantization method RQ [19], DBQ drastically improves the Top-1 accuracy of MobileNetV1 on ImageNet from $61.50 \%$ to $70.92 \%$ at iso-model size accompanied by a $19 \%$ reduction in computational complexity.

4. For lightweight networks tackling real world applications, we show that DBQ with two ternary branches offers the best (pareto-optimal) accuracycomplexity trade-off compared to using one ternary branch with higher number of channels, at iso-model size.

\section{Related Work}

Reducing DNN complexity via quantization has been an active area of research over the past few years. A majority of such works either train the quantized network from scratch $[36,33,16,10,22,24]$ or fine-tune a pre-trained model with quantization-in-the-loop $[13,19,30,32,1,35]$. Where retraining is not an option, [25] provides analytical guarantees on the minimum precision requirements of a pre-trained FP network given a budget on the accuracy drop from FP. Training based quantization works fall into two classes of methods: 1) estimation based methods [33,18,16,30,13], where the full-precision weights and activations are quantized in the forward path, and gradients are back-propagated through a nondifferentiable quantizer function via a gradient estimator such as the Straight Through Estimator (STE) [2]; and 2) optimization based methods, where gradients flow directly from the full-precision weights to the cost function via an approximate differentiable quantizer $[32,19,24]$, or by including an explicit quantization error term to the loss function $[7,35]$. Application of these methods can be categorized into three clusters: 
Aggressive Quantization: Methods such as binarization and ternarization have been highly successful for reducing DNN complexity. BinaryNets [10] quantize both weights and activations of DNNs to \pm 1 , while XNORNets [22] use a fullprecision scalar to represent binarized weights in order to improve accuracy. Ternary Weight Networks (TWN) [16] quantize weights to $\{-1,0,1\}$ and leverage the resulting weight sparsity due to the '0' state to skip operations. Trained Ternary Quantization (TTQ) [36] proposes learning the ternary scales via backprop. However, a major drawback of such methods is the resulting accuracy loss especially when applied to lightweight

\begin{tabular}{|c|c|c|c|}
\hline Layer $T$ & Iults [? & Adds & Params \\
\hline$\overline{F L}$ & 1.89 & 1.83 & 0.02 \\
\hline DW & 3.03 & 2.72 & 1.05 \\
\hline $\mathrm{PW}$ & 94.02 & 94.37 & 74.19 \\
\hline $\mathrm{FC}$ & 0.18 & 0.18 & 24.22 \\
\hline $\mathrm{PL}$ & 0 & 0.01 & 0 \\
\hline BN & 0.88 & 0.89 & 0.52 \\
\hline
\end{tabular}

Table 1. The number of multiplications, additions, and parameters required by each layer type: first layer (FL), depthwise (DW), pointwise (PW), fully connected (FC), pooling layer (PL), and batch normalization $(\mathrm{BN})$, for a single inference using MobileNetV1.

DNNs such as MobileNet. In Section 4.3,

we show that ternarizing only the pointwise layers in MobileNetV1 on Imagenet, which correspond to $\sim 94 \%$ of the total multiplication/additions (Table 1), incurs a massive accuracy loss $(\sim 5.67 \%)$ compared to the full-precision baseline. Hence, such methods are typically benchmarked on simple datasets such as CIFAR-10, or use over parameterized models such as AlexNet [15] or ResNet-18 [6] on ImageNet. In contrast, our proposed DBQ method is able to aggressively quantize the lightweight MobileNetV1 architecture with minimal loss in Top-1 accuracy (Fig 1).

Non-uniform Quantization: These methods seek to improve the performance of binarized/ternarized models while leveraging their arithmetic simplicity, e.g., LQNets [33] and ABCNets [18], by quantizing weights and activations as linear combinations of binary values. The resulting non-uniform multi-bit quantization allows the computation of dot products to be carried out using binary arithmetic with appropriate scaling and addition. However, these methods suffer from two major drawbacks: 1) the design of their quantization functions is computationally expensive as it requires an iterative solution of a non-convex optimization problem per-layer per-forward pass during training, which results in a significant training time overhead in the range $1.4 \times-3.7 \times[33]$; and 2 ) they suffer from gradient mismatch problems as they depend on the STE [2] method to compute the gradients during training. This renders the quantizer constructed by these methods to be sub-optimal, since they estimate the quantizer parameters by minimizing a local cost function, e.g., MSE. Moreover, these methods have been benchmarked only on over parameterized networks on ImageNet. Whereas our proposed DBQ method learns the multiple ternary branches by minimizing a global loss function since the proposed quantizer is fully differentiable, which enables the efficient training of similar non-uniform quantizers, while also eradicating the need for any gradient estimator. 
Quantization of Lightweight DNNs: Recent works that quantize MobileNets either apply fixed-point quantization with uniform $[19,28,13]$ or mixed [30,29] precision across layers. Hardware-Aware Quantization (HAQ) [30] proposes using reinforcement learning to learn the per-layer bit-precision for both weights and activations, whereas [29] learns the bit-precision via a reformulation of the quantizer function and relying on the STE for gradient computation. Integer-Arithmetic-Only (IAO) [13] proposes using 8-b quantization for accelerating the inference of MobileNets on hardware platforms such as Qualcom Hexagon and ARM NEON. Relaxed Quantization (RQ) [19] approximates the quantization function with a smooth differentiable approximate function, but the quantized values are still in fixed-point. Uniform Noise Injection Quantization (UNIQ) [1] proposes training a non-uniform quantizer using a special noise injection method that allows natural computation of gradients for quantized parameters. UNIQ uses a non-uniform quantizer requiring inefficient lookup tables and full precision multipliers/additions. Furthermore, all of these approaches use relatively high bitwidths $(\sim 6 \mathrm{~b}-8 \mathrm{~b})$, and most even fail to bridge the accuracy gap between the quantized models and their full-precision baseline. In contrast, the proposed DBQ method is able to aggressively reduce the precision of the dominant (94\%) PW layers of MobileNetV1 to two ternary parameters with negligible degradation in the Top-1 accuracy.

\section{$3 \quad$ Differentiable Branched Quantizer (DBQ)}

A ternary $B$-branch quantizer $Q(\mathbf{w})$ of a full precision weight vector $\mathbf{w} \in \mathbb{R}^{D}$ (Fig 2(a)) is given by:

$$
\mathbf{w}_{q}=Q(\mathbf{w})=\sum_{j=1}^{B} \alpha_{j} \mathbf{w}_{j}
$$

where $\mathbf{w}_{j} \in\{-1,0,1\}^{D}$ are the ternary branch weight vectors, and $\forall j \in[B]$ : $\alpha_{j}>0$ are per-branch scalars. In DBQ, we wish to learn all the network parameters which requires the quantizer function $Q(\mathbf{w})$ to be made differentiable. To do so, we first formulate a parametric form of $Q(\mathbf{w})$ in Section 3.1 and then employ a smooth 'temperature-controlled' approximation of the quantizer step function to establish its differentiability in Section 3.2.

\subsection{Formulation of DBQ}

We formulate the ternary $B$-branch quantizer in Fig. 2 as a $N=3^{B}$-level nonuniform quantizer $Q(\mathbf{w}): \mathbb{R}^{D} \rightarrow \mathcal{V}^{D}$ with quantization levels $\mathcal{V}=\left\{v_{i}\right\}_{i=1}^{N}$. Assuming that the quantization levels $v_{i}$ 's are sorted in ascending order, the $Q(\mathbf{w})$ can be written as a linear combination of $N-1$ step functions as shown below:

$$
Q(\mathbf{w})=\sum_{i=1}^{N-1}\left[\left(v_{i+1}-v_{i}\right) f\left(\mathbf{w}-t_{i}\right)\right]-\frac{v_{N}-v_{1}}{2}
$$




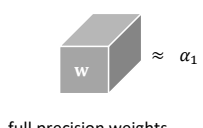

full precision weights

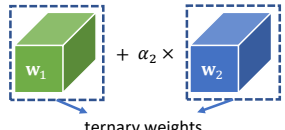

(a)

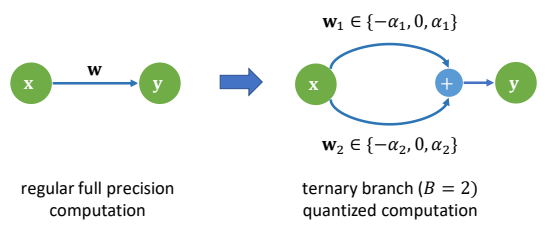

(b)

Fig. 2. Branched quantization of full precision weights: (a) as a linear combination of ternary weights, and (b) implemented as multiple parallel ternary branch operations to leverage the properties of ternary arithmetic for dot product computations.

where $f(\mathbf{u})=\left[\mathbf{1}_{\left\{u_{1}>0\right\}}, \ldots, \mathbf{1}_{\left\{u_{D}>0\right\}}\right]^{\mathrm{T}}$ is an element-wise ideal step function, and $\left\{t_{i}\right\}_{i=1}^{N-1}$ are the quantizer thresholds. The $\left(v_{N}-v_{1}\right) / 2$ term is the quantizer offset. We impose the ternary quantizer structure in (1) via the constraint:

$$
v_{i}=\sum_{j=1}^{B} e_{i, j} \alpha_{j}
$$

where $e_{i, j} \in\{-1,0,1\}$, and thereby obtain the final quantizer expression:

$$
Q(\mathbf{w})=\gamma_{2}\left[\sum_{i=1}^{N-1}\left[f\left(\gamma_{1} \mathbf{w}-t_{i}\right) \sum_{j=1}^{B} b_{i, j} \alpha_{j}\right]-\sum_{j=1}^{B} \alpha_{j}\right]
$$

where $b_{i, j}=e_{i+1, j}-e_{i, j} \in\{-2,-1,0,1,2\} \forall j \in[B]$ are fixed coefficients, and $\gamma_{1} \& \gamma_{2}$ are pre/post-quantization scales to ensure that the quantizer operates on normalized inputs. Thus, the branched quantizer is parametrized by $\mathcal{P}_{Q}=$ $\left\{\alpha_{1}, \ldots, \alpha_{B}, \gamma_{1}, \gamma_{2}, t_{1}, \ldots, t_{N-1}\right\}$ and these all need to be learned.

In this paper, we focus on the $B=2$ case, i.e., two ternary branch, as visualized in Fig. 3, with $N=3^{2}=9$ different quantization levels $v_{i}$. In this case, (4) can be expanded as:

$$
\begin{aligned}
Q(\mathbf{w}) & =\gamma_{2}\left[\alpha_{2} f\left(\gamma_{1} \mathbf{w}-t_{1}\right)+\left(\alpha_{1}-\alpha_{2}\right) f\left(\gamma_{1} \mathbf{w}-t_{2}\right)+\left(2 \alpha_{2}-\alpha_{1}\right) f\left(\gamma_{1} \mathbf{w}-t_{3}\right)\right. \\
& +\left(\alpha_{1}-\alpha_{2}\right) f\left(\gamma_{1} \mathbf{w}-t_{4}\right)+\left(\alpha_{1}-\alpha_{2}\right) f\left(\gamma_{1} \mathbf{w}-t_{5}\right)+\left(2 \alpha_{2}-\alpha_{1}\right) f\left(\gamma_{1} \mathbf{w}-t_{6}\right) \\
& \left.+\left(\alpha_{1}-\alpha_{2}\right) f\left(\gamma_{1} \mathbf{w}-t_{7}\right)+\alpha_{2} f\left(\gamma_{1} \mathbf{w}-t_{8}\right)-\left(\alpha_{1}+\alpha_{2}\right)\right]
\end{aligned}
$$

\subsection{Differentiability}

Inspired by $[32,31]$, we replace the non-differentiable $f$ in (4) with a smooth sigmoid approximation $\hat{f}_{T}$ as follows:

$$
\hat{f}_{T}(u)=\frac{1}{1+\exp (-T u)}
$$




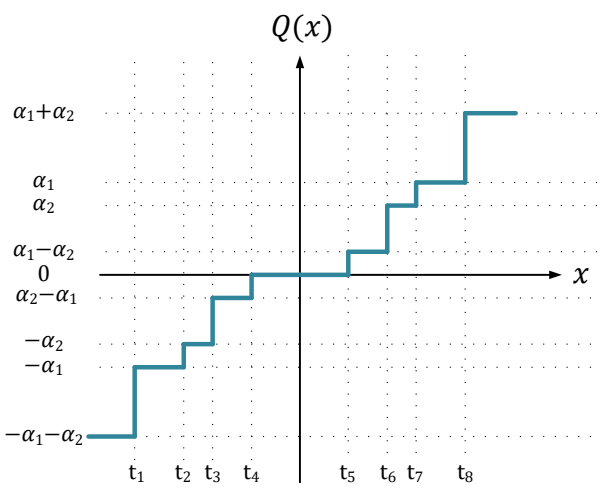

Fig. 3. Visualization of a two ternary (2T) branch quantizer with branch scales $\alpha_{1}$ and $\alpha_{2}$ assuming $\alpha_{1} \geq \alpha_{2} \geq \frac{\alpha_{1}}{2} \geq 0$.

where the temperature parameter $T$ controls the approximation error, i.e.,:

$$
e_{T}(u)=\hat{f}_{T}(u)-f(u) \underset{T \rightarrow \infty}{\longrightarrow} 0
$$

When learning the quantizer parameters $\mathcal{P}_{Q}$, the temperature $T$ is increased gradually as the training converges so that $\hat{f}_{T}(u) \rightarrow f(u)$. The resultant differentiable quantizer $Q_{T}(\mathbf{w})=\mathbf{w}_{q}=\mathbf{z}$ therefore enables a straightforward calculation of the gradients for all quantizer and model parameters w.r.t. loss function $\mathcal{L}$ as follows:

$$
\begin{aligned}
\frac{\partial \mathcal{L}}{\partial \gamma_{2}} & =\frac{1}{\gamma_{2}} \sum_{k=1}^{D} \frac{\partial \mathcal{L}}{\partial z_{k}} z_{k} \\
\frac{\partial \mathcal{L}}{\partial \alpha_{j}} & =\gamma_{2} \sum_{k=1}^{D} \frac{\partial \mathcal{L}}{\partial z_{k}}\left[\sum_{i=1}^{N-1}\left[b_{i, j} g_{k, i}\right]-1\right] \\
\frac{\partial \mathcal{L}}{\partial t_{i}} & =-\gamma_{2} T \sum_{k=1}^{D} \frac{\partial \mathcal{L}}{\partial z_{k}}\left[h_{k, i} \sum_{j=1}^{B} b_{i, j} \alpha_{j}\right] \\
\frac{\partial \mathcal{L}}{\partial w_{k}} & =\gamma_{1} \gamma_{2} T \frac{\partial \mathcal{L}}{\partial z_{k}} \sum_{i=1}^{N-1}\left[h_{k, i} \sum_{j=1}^{B} b_{i, j} \alpha_{j}\right] \\
\frac{\partial \mathcal{L}}{\partial \gamma_{1}} & =\gamma_{2} T \sum_{k=1}^{D} \frac{\partial \mathcal{L}}{\partial z_{k}} w_{k}\left[\sum_{i=1}^{N-1}\left[h_{k, i} \sum_{j=1}^{B} b_{i, j} \alpha_{j}\right]\right]
\end{aligned}
$$

where $h_{k, i}=g_{k, i}\left(1-g_{k, i}\right)$ and $g_{k, i}=\hat{f}_{T}\left(\gamma_{1} w_{k}-t_{i}\right)$ for brevity. By doing so, we eliminate the need for the STE and the expensive computational overhead introduced in estimation-based methods such as LQNet [33] or ABCNet [18]. Note that software frameworks such as PyTorch [21] automatically take care of computing these gradients so these don't need to be explicitly coded. 


\subsection{Implementation Details}

Parameter Initialization: Initializing the quantizer parameters $\mathcal{P}_{Q}$ is performed once before training and requires an initial vector $\mathbf{w} \in \mathbb{R}^{D}$ which can be from a pre-trained network or from random initialization (training from scratch). The initialization procedure is as follows: 1) the post-quantization scale $\gamma_{2}$ is set to the maximum absolute value in $\mathbf{w}$, and the pre-quantization scale $\gamma_{1}$ is set to $1 / \gamma_{2}$. This ensures that the quantizer operates on normalized parameters which facilitates the optimization of its parameters, and that the quantized values are of the same scale as the inputs; 2) to find the optimal thresholds $\left\{t_{i}\right\}_{i=1}^{N-1}$, we first compute the optimal $N$ centroids $\left\{c_{i}\right\}_{i=1}^{N}$ of the normalized vector $\gamma_{1} \mathbf{w}$ via $k$-means, and then $\forall i \in[N-1]$ we set $t_{i}$ to be the midpoint of the interval $\left[c_{i}, c_{i+1}\right]$; and 3) a good initialization for $\left\{\alpha_{j}\right\}_{j=1}^{B}$ is found by solving for the optimal values that minimize the $L_{2}$ norm between the normalized vector $\gamma_{1} \mathbf{w}$ and its quantized counterpart.

Training and Inference: During training, the proposed DBQ quantizer is used with the approximate smooth step function $\hat{f}_{T}$ for both forward and backward calculations $((4) \&(8)-(12))$. For a given layer in the network that performs the function $\mathbf{y}=F(\mathbf{w}, \mathbf{x})$, applying DBQ simply boils down to composing the quantizer described in (4) with the function $F: \mathbf{y}=F\left(Q_{T}(\mathbf{w}), \mathbf{x}\right)$. For quantizing convolutional layers, we apply kernel-wise quantizers. The overhead of full precision scales is amortized across the large filter lengths. The choice of the temperature parameter $T$ is important. A large value of $T$ would reduce the approximation error in (7), however the gradients would saturate quickly, thus causing a bottleneck for learning the quantizer parameters. Therefore, an initial small value for $T$ is used for the first training epoch, and its value is increased for successive epochs based on a pre-determined temperature update schedule. A simple yet effective schedule is to linearly increment the temperature with the number of epochs: $T=T_{\text {init }}+e \times T_{\mathrm{inc}}$. During inference, the approximate step function is replaced with the ideal function $f$ such that the quantizer output satisfies (1).

Activation Quantization: The challenge in quantizing input activations with a fixed-point quantizer during training is determining a suitable clipping value (Fig. 4). Traditionally, the use of ReLU6 (which clips at 6) has been a popular choice due to its simplicity $[28,13]$. However, the choice of 6 provides no guarantees on the clipping probability, and can therefore yield sub-optimal results. Similar to [4], we propose clipping the post-BN activations $y_{\mathrm{BN}}$ (Fig. 4) using:

$$
c=\max _{i \in[C]}\left(\beta_{l}^{(i)}+k \gamma_{l}^{(i)}\right)
$$

where $C$ is the number of channels in the activation tensor $y_{1},\left(\beta^{(i)}, \gamma^{(i)}\right)$ are learnable per-channel shift and scale parameters of $\mathrm{BN}$, and $k$ is a network hyperparameter that controls the clipping probability. Assuming that the distribution of $y_{\mathrm{BN}}^{(i)} \sim \mathcal{N}\left(\beta^{(i)},\left(\gamma^{(i)}\right)^{2}\right)$ [12] and using $6 \sigma$ rule $(k=6)$, one can show that the choice of $c$ in (13) guarantees:

$$
\operatorname{Pr}\left\{y_{\mathrm{BN}} \leq c\right\} \geq 0.999
$$




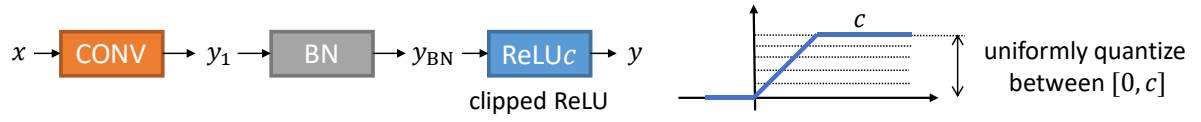

Fig. 4. Quantizing activations post-ReLU requires a pre-determined clipping parameter c.

Note that having a fixed clipping value $c$ for all channels is crucial in order to ensure that the dot product operations can be implemented in fixed-point.

\section{Experimental Results}

To demonstrate the effectiveness of the DBQ method for quantizing lightweight networks, we evaluate it on three different image classification datasets: 1) CIFAR10 [14] using ResNet-20 [6]; 2) ImageNet (ILSVRC 2012) [23] using MobileNetV1 [8], MobileNetV2 [27], and ShuffleNetV2 [20]; and 3) the recently proposed Visual Wake Words [3] using MobileNetV1. In all of our experiments, we train full precision models from scratch, and perform fine tuning on said models for training their quantized counterparts. We use stochastic gradient descent for training all the models. For further details on the training setup for each experiment, please check the supplementary material.

\subsection{Complexity Metrics}

We propose a set of metrics, inspired by those used in [25,26], in order to quantify the complexity reduction achieved by our proposed method.

Computational Cost $\left(\mathcal{C}_{\boldsymbol{C}}\right)$ for an $L$-layer network:

$$
\mathcal{C}_{C}=\sum_{l=1}^{L} N_{l}\left[D_{l} B_{W, l} B_{A, l}+\left(D_{l}-1\right)\left(B_{A, l}+B_{W, l}+\left\lceil\log _{2} D_{l}\right\rceil-1\right)\right]
$$

where $N_{l}$ is the number of $D_{l}$-dimensional dot products in layer $l$ with $B_{W, l}$ and $B_{A, l}$ being the weights and activations precisions respectively. This cost essentially measures the number of $1 \mathrm{~b}$ full adders (FAs) needed to implement the dot products required for a given network. For full precision (32b) parameters, we make the simplifying assumption of treating them as $23 \mathrm{~b}$ (mantissa precision) fixed-point parameters.

Sparsity-Aware Computational Cost $\left(\mathcal{C}_{\boldsymbol{S}}\right)$ is also defined in order to leverage weight-sparsity in different models that can be reflected on the model complexity:

$$
\mathcal{C}_{S}=\sum_{l=1}^{L} N_{l}\left[D_{l}^{\prime} B_{W, l} B_{A, l}+\left(D_{l}^{\prime}-1\right)\left(B_{A, l}+B_{W, l}+\left\lceil\log _{2} D_{l}\right\rceil-1\right)\right]
$$




\begin{tabular}{llcc}
\hline Method & Acc. $(\boldsymbol{\Delta})[\%]$ & $\mathcal{C}_{\boldsymbol{C}}\left(\mathcal{C}_{\boldsymbol{S}}\right)\left[10^{9} \mathrm{FA}\right] \mathcal{C}_{\boldsymbol{R}}\left(\mathcal{C}_{\boldsymbol{M}}\right)\left[10^{6} \mathrm{~b}\right]$ \\
\hline FP [33] & $92.10(/)$ & $23.73(23.73)$ & $14.63(8.63)$ \\
\hline LQNet-1B [33] & $90.10(-2.171)$ & $1.60(1.60)$ & $6.34(0.35)$ \\
\hline LQNet-2B [33] & $91.80(-0.325)$ & $2.83(2.83)$ & $6.61(0.61)$ \\
\hline LQNet-3B [33] & $92.00(-0.108)$ & $4.07(4.07)$ & $6.88(0.88)$ \\
\hline \hline FP (Ours) & $92.00(/)$ & $23.73(23.73)$ & $14.63(8.63)$ \\
\hline DBQ-1T (Ours) & $\mathbf{9 1 . 0 6 ( - \mathbf { 1 . 0 2 1 } )}$ & $\mathbf{1 . 6 0}(\mathbf{0 . 9 2})$ & $6.61(0.61)$ \\
\hline DBQ-2T (Ours) & $\mathbf{9 1 . 9 3 ( - \mathbf { 0 . 0 7 6 } )}$ & $\mathbf{2 . 8 3 ( \mathbf { 1 . 7 9 } )}$ & $7.15(1.15)$ \\
\hline
\end{tabular}

Table 2. The accuracy on CIFAR-10 and complexity metrics $\left(\mathcal{C}_{C}, \mathcal{C}_{S}, \mathcal{C}_{R}, \mathcal{C}_{M}\right)$ for ResNet-20 using our method DBQ compared to LQNet. $\Delta$ represents the normalized accuracy drop of the quantized models with respect to its full precision baseline. The first, last layers, and input activations are kept in full precision for the quantized models in accordance with [33].

where $D_{l}^{\prime}$ is the number of non-zero weights in the corresponding $D_{l}$-dimensional dot product.

Representational Cost $\left(\mathcal{C}_{\boldsymbol{R}}\right)$ for an $L$-layer network:

$$
\mathcal{C}_{R}=\sum_{l=1}^{L}\left[\left|W_{l}\right| B_{W, l}+\left|A_{l}\right| B_{A, l}\right]
$$

where $\left|W_{l}\right|$ and $\left|A_{l}\right|$ are the number of elements in the weight and activation tensors in layer $l$, respectively.

Model Storage Cost $\left(\mathcal{C}_{M}\right)$ for an $L$-layer network:

$$
\mathcal{C}_{M}=\sum_{l=1}^{L}\left|W_{l}\right| B_{W, l}
$$

which only accounts for the weight storage, and can be useful for studying model compression.

\subsection{CIFAR-10 Results}

We first demonstrate the effectiveness of DBQ on the CIFAR-10 dataset using the popular network ResNet-20 [6]. To ensure a fair comparison with the LQNet [33] models, we do not quantize the first and last fully connected layers, and we keep all activations in full precision. Table 2 summarizes the accuracy (and percentage drop) as well as the four complexity metrics $\left(\mathcal{C}_{C}, \mathcal{C}_{S}, \mathcal{C}_{R}, \mathcal{C}_{M}\right)$ for different number of branches used. At iso-number of branches, the DBQ models achieve higher accuracies for the same $\mathcal{C}_{C}$ and lower $\mathcal{C}_{S}$ due to the high number of zero valued weights, as opposed to binary branches where the weights are either \pm 1 . Comparing the DBQ-2T and LQNet-3B models, which achieve comparable accuracies, DBQ-2T requires $\sim 32 \%$ less $\mathcal{C}_{C}$ and $\sim 56 \%$ less $\mathcal{C}_{S}$, at the expense of an extra bit per-parameter, which is reflected in the marginal $\sim 4 \%$ increase in $\mathcal{C}_{R}$. 


\begin{tabular}{l|ccccc|ccc}
\hline Model Name & Activations FL DW PW & FC & Top-1/5 Acc. $[\%]$ & $\mathcal{C}_{\boldsymbol{C}}\left(\mathcal{C}_{\boldsymbol{S}}\right)\left[10^{10} \mathrm{FA}\right]$ & $\mathcal{C}_{\boldsymbol{R}}\left(\mathcal{C}_{\boldsymbol{M}}\right)\left[10^{7} \mathrm{~b}\right]$ \\
\hline FP & ReLU - 32b & 32b & 32b & 32b & 32b & $\mathbf{7 2 . 1 2 / 9 0 . 4 3}$ & $33.37(33.37)$ & $30.00(13.54)$ \\
\hline FX8-1 & ReLU6 - 8b & 32b & 8b & 8b & 32b & $71.65 / 90.17$ & $5.78(5.39)$ & $10.38(5.90)$ \\
\hline FX8-2 & ReLU6 - 8b & 8b & 8b & 8b & 8b & $71.60 / 90.19$ & $5.24(4.85)$ & $7.56(3.44)$ \\
\hline FX8-3 & ReLU $x$ - 8b & 8b & 8b & 8b & 8b & $\mathbf{7 1 . 8 6 / 9 0 . 2 6}$ & $5.24(4.85)$ & $7.56(3.44)$ \\
\hline DBQ-1T & ReLU - 32b & 32b & 32b & 1T & 32b & $66.45 / 86.72$ & $3.60(2.61)$ & $20.58(4.12)$ \\
\hline DBQ-2T-1 & ReLU - 32b & 32b & 32b & 2T & 32b & $71.09 / 89.71$ & $5.23(3.77)$ & $21.21(4.75)$ \\
\hline DBQ-2T-2 & ReLU6 - 8b & 32b & 8b & 2T & 32b & $70.25 / 89.42$ & $2.73(1.97)$ & $9.12(4.64)$ \\
\hline DBQ-2T-3 & ReLU $x-$ 8b & 32b & 8b & 2T & 32b & $70.80 / 89.75$ & $2.73(1.97)$ & $9.12(4.64)$ \\
\hline DBQ-2T-4 & ReLU $x-$ 8b & 8b & 8b & 2T & 8b & $\mathbf{7 0 . 9 2 / 8 9 . 6 1}$ & $\mathbf{2 . 1 8}(\mathbf{1 . 4 2})$ & $\mathbf{6 . 3 0}(\mathbf{2 . 1 8})$ \\
\hline
\end{tabular}

Table 3. The Top- $1 / 5$ accuracy on ImageNet and complexity metrics $\left(\mathcal{C}_{C}, \mathcal{C}_{S}, \mathcal{C}_{R}, \mathcal{C}_{M}\right)$ for MobileNetV1 under different precision assignments. Models denoted by DBQ- $z \mathrm{~T}$ are trained using our differentiable branch quantizer with $B=z$ ternary branches. ReLUx denotes a clipped ReLU using our proposed clipping method in Eq. (13).

\subsection{ImageNet Results}

In this section, we report results for MobileNetV1 [8], MobileNetV2 [27], and ShuffleNetV2 [20] on ImageNet. We first focus on MobileNetV1 by performing an ablation study, and leverage these results for quantizing the more recent MobileNetV2 and ShuffleNetV2.

Ablation Study: Table 3 summarizes the Top-1,5 accuracies of all the MobileNetV1 models trained with different layer precision assignments in order to evaluate the impact of our design choices. To see the impact of using two ternary branches instead of one, we begin with the DBQ-1T model which is obtained by quantizing only the PW layers of MobileNetV1 to one ternary branch (1T) keeping all other activations and weights in full precision. Table 3 shows that DBQ-1T achieves a massive $89 \%$ reduction in $\mathcal{C}_{C}$ compared to the $\mathrm{FP}$ model but at a catastrophic loss of $5.67 \%$ in the Top-1 accuracy. In contrast, DBQ-2T-1, which is DBQ-1T with a second ternary branch, is able to recover accuracy to within $1.03 \%$ of the full-precision baseline while also achieving massive savings in $\mathcal{C}_{C}$ of $84 \%$. Quantizing the activations and the remaining layers weights of DBQ-2T-1 to $8 \mathrm{~b}$ fixed-point, i.e., DBQ-2T-4, incurs a minimal loss in accuracy of $1.2 \%$ compared to the FP model while also achieving even greater reduction in both $\mathcal{C}_{C}(93 \%)$ and $\mathcal{C}_{R}(70 \%)$. The reduction in $\mathcal{C}_{S}$ increases to $96 \%$ when branch sparsity is exploited to skip computations.

Note that the reason that only PW layers are quantized using ternary branches is three-fold: 1) PW layers consume $\sim 94 \%$ of the amount of multiply-adds required for inference (Table 1); 2) we have observed that quantizing the PW layers has the most severe impact on classification accuracy compared to quantizing other layers; and 3) DW layers suffer from extremely small dot-product lengths (9), rendering them unsuitable for multiple branch quantization (the overhead of branch-merge and scaling operations will dominate).

The benefits of our proposed BN-based clipping described in (13) can be seen by comparing the accuracy of the $8 \mathrm{~b}$ fixed-point model FX8-3 using BN-based clipping with $k=6$ with its ReLU6-based clipping counterpart FX8-2. The Top- 


\begin{tabular}{|c|c|c|c|c|c|c|c|c|}
\hline Method & Act. & $\overline{\text { FL }}$ & DW & $\overline{\text { PW }}$ & FC & Top-1 Acc. $[\%]$ & $\mathcal{C}_{C}\left(\mathcal{C}_{S}\right)\left[10^{10} \mathrm{FA}\right]$ & $\mathcal{C}_{R}\left(\mathcal{C}_{M}\right)\left[10^{7} \mathrm{~b}\right]$ \\
\hline$\overline{\mathrm{IAO}^{\star}[13]}$ & $8 \mathrm{~b}$ & $8 \mathrm{~b}$ & $8 \mathrm{~b}$ & $8 \mathrm{~b}$ & $8 \mathrm{~b}$ & $69.00^{*}$ & $4.97(/)$ & $7.49(3.37)$ \\
\hline UNIQ [1] & $8 \mathrm{~b}$ & $5 b$ & $5 \mathrm{~b}$ & $5 b$ & $5 b$ & 67.50 & $3.70(/)$ & $6.29(2.18)$ \\
\hline UNIQ [1] & $8 \mathrm{~b}$ & $4 \mathrm{~b}$ & $4 \mathrm{~b}$ & $4 \mathrm{~b}$ & $4 \mathrm{~b}$ & 66.00 & $3.19(/)$ & $5.87(\mathbf{1 . 7 6})$ \\
\hline UNIQ [1] & $8 \mathrm{~b}$ & $8 \mathrm{~b}$ & $8 \mathrm{~b}$ & $8 \mathrm{~b}$ & $8 \mathrm{~b}$ & 68.25 & $5.24(/)$ & $7.56(3.44)$ \\
\hline $\mathrm{QSM}^{\star}[28]$ & $8 \mathrm{~b}$ & $8 \mathrm{~b}$ & $8 \mathrm{~b}$ & $8 \mathrm{~b}$ & $8 \mathrm{~b}$ & 68.03 & $4.97(/)$ & $7.49(3.37)$ \\
\hline RQ [19] & $5 \mathrm{~b}$ & $5 b$ & $5 \mathrm{~b}$ & $5 \mathrm{~b}$ & $5 \mathrm{~b}$ & 61.50 & $2.68(/)$ & $4.75(2.18)$ \\
\hline RQ [19] & $6 \mathrm{~b}$ & $6 \mathrm{~b}$ & $6 \mathrm{~b}$ & $6 \mathrm{~b}$ & $6 \mathrm{~b}$ & 67.50 & $3.42(/)$ & $5.69(2.60)$ \\
\hline HAQ cloud [30] & mixed & $8 \mathrm{~b}$ & mixed & mixed & $8 \mathrm{~b}$ & $65.33-71.20^{\dagger}$ & $2.73(/)$ & $5.09(3.12)$ \\
\hline HAQ edge [30] & mixed & $8 \mathrm{~b}$ & mixed & mixed & $8 \mathrm{~b}$ & $67.40-71.20^{\dagger}$ & $4.06(/)$ & $5.87(2.49)$ \\
\hline FX8 (Ours) & $8 \mathrm{~b}$ & $8 \mathrm{~b}$ & $8 \mathrm{~b}$ & $8 \mathrm{~b}$ & $8 \mathrm{~b}$ & 71.86 & $5.24(4.85)$ & $7.56(3.44)$ \\
\hline DBQ-2T (Ours) & $8 \mathrm{~b}$ & $8 \mathrm{~b}$ & $8 \mathrm{~b}$ & $2 \mathrm{~T}$ & $8 \mathrm{~b}$ & 70.92 & $2.18(1.42)$ & $6.30(2.18)$ \\
\hline
\end{tabular}

Table 4. The Top-1 accuracy on ImageNet and complexity metrics $\left(\mathcal{C}_{C}, \mathcal{C}_{S}, \mathcal{C}_{R}, \mathcal{C}_{M}\right)$ for MobileNetV1 using our method (DBQ-2T) compared to state-of-the art training-based quantization methods.

1 accuracy of FX8-3 is better than FX8-2 without any overhead in training or inference. Similarly for DBQ-2T-3 and DBQ-2T-2.

Branching Utility: A $2 \mathrm{~T}$ quantizer should result in 9 distinct quantization levels as shown in Fig. 3. However, in a $2 \mathrm{~T}$ branched quantizer such as ours, it is possible for the number of quantization levels to be smaller than 9 , e.g., if $\alpha_{1}=\alpha_{2}$ then the number of quantization levels is 5 . In this case, the full representational power of the $2 \mathrm{~T}$ branched quantizer is not utilized. To see if the $2 \mathrm{~T}$ branched quantizer generates all 9 levels, we plot the distribution of the ratio $R_{\alpha}=\frac{\alpha_{1}}{\alpha_{2}}$ across all the PW layers in the DBQ-2T-4 model (Table 3). The distribution is

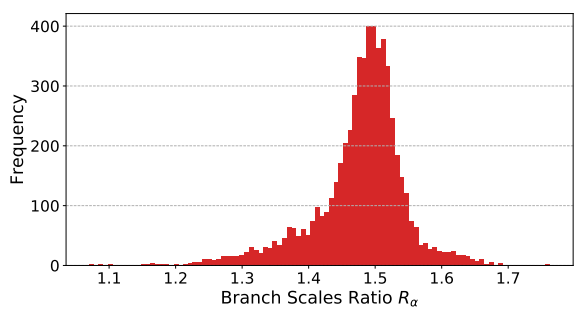
centered around $R_{\alpha}=1.48$ with more

Fig. 5. The distribution of the ratio of the ternary branch scales $\alpha_{1}$ and $\alpha_{2}$ for DBQ2T-4 from Table 3. than $99 \%$ of the values lying in the range $[1.2,1.7]$. This demonstrates that the quantizer learned by DBQ employs the full representational power offered by the $2 \mathrm{~T}$ structure.

Comparison with State-of-the Art: Table 4 compares the performance of our proposed DBQ method against state-of-the art results on ImageNet for MobileNetV1. Our model DBQ-2T, which corresponds to DBQ-2T-4 in Table 3 achieves the lowest computational cost $\mathcal{C}_{C}\left(2.18 \times 10^{10} \mathrm{FAs}\right)$ compared to previously published networks, while achieving the highest Top-1 accuracy $70.92 \%$. Compared to the lowest complexity model RQ [19], DBQ-2T achieves a $19 \%$ reduction in $\mathcal{C}_{C}$ with a $9.42 \%$ improvement in Top-1 accuracy at iso-storage 


\begin{tabular}{|c|c|c|c|c|}
\hline Model & Act. FL DW PW FC & Top-1 Acc. $[\%$ & $\mathcal{C}_{C}\left(\mathcal{C}_{S}\right)\left[10^{10} \mathrm{FA}\right]$ & $\mathcal{C}_{\boldsymbol{R}}\left(\mathcal{C}_{M}\right)\left[10^{7} \mathrm{~b}\right]$ \\
\hline MobileNetV2-FP & $\begin{array}{llllll}2 \mathrm{~b} & 32 \mathrm{~b} & 32 \mathrm{~b} & 32 \mathrm{~b} & 32 \mathrm{~b}\end{array}$ & 71.88 & $17.83(17.83)$ & $32.87(11.22)$ \\
\hline MobileNetV2-2T & $2 \mathrm{~T} \quad 8 \mathrm{~b}$ & 70.54 & $1.42(1.11)$ & $7.45(2.04)$ \\
\hline ShuffleNetV2-FP & $\begin{array}{lllll}32 \mathrm{~b} & 32 \mathrm{~b} & 32 \mathrm{~b} & 32 \mathrm{~b} & 32 \mathrm{~b}\end{array}$ & 69.36 & $8.52(8.52)$ & $13.81(7.29)$ \\
\hline ShuffleNetV2-2T & $\begin{array}{lllll}8 \mathrm{~b} & 8 \mathrm{~b} & 8 \mathrm{~b} & 2 \mathrm{~T} & 8 \mathrm{~b}\end{array}$ & 66.74 & $0.64(0.46)$ & $3.21(1.38)$ \\
\hline
\end{tabular}

Table 5. The Top-1 accuracy on ImageNet and complexity metrics $\left(\mathcal{C}_{C}, \mathcal{C}_{S}, \mathcal{C}_{R}, \mathcal{C}_{M}\right)$ for MobileNetV2 and ShuffleNetV2 using our method (DBQ-2T).

complexity $\mathcal{C}_{M}$. Furthermore, DBQ-2T improves upon the accuracy of the IAO model [13], which currently achieves the highest Top-1 accuracy, by $1.92 \%$ but with a massive reduction in complexity $\mathcal{C}_{C}(56 \%), \mathcal{C}_{R}(16 \%)$, and $\mathcal{C}_{M}(35 \%)$.

More Lightweight Networks: Table 5 demonstrates the performance of DBQ when applied to the more recent lightweight networks: MobileNetV2 and ShuffleNetV2. Similar to MobileNetV1, we find that the PW layers dominate the number of operations required for a single inference for both MobileNetV2 $(87 \%)$ and ShuffleNetV2 (90\%). Thus, and inline with our experiments on MobileNetV1, we quantize all PW layers using $2 \mathrm{~T}$, with the remaining layers and activations quantized to 8b fixed-point. We observe a minimal 1.3\% (MobileNetV2) and $2.6 \%$ (ShuffleNetV2) drop in accuracy compared to FP, while achieving massive $(77 \%-95 \%)$ reductions in all the complexity metrics. A comparison between DBQ and [29] for MobileNetV2 is present in the supplementary material.

\subsection{Visual Wake Words Results}

We study the accuracy-precision-complexity trade-off in quantized DNNs using the Visual Wake Words (VWW) dataset that was recently proposed by Google [3] in order to facilitate the development of lightweight vision models for deployment on resource-constrained Edge devices. This dataset reflects a typical real-world scenario involving the detection of specific events by observing incoming data, e.g., monitoring a camera video feed in order to detect the presence of a person [3], similar to the use of audio wake words in speech recognition. The VWW dataset is derived from the COCO dataset [17] via a simple re-labeling of the available images has a training set of $115 \mathrm{k}$ images and a test set with $8 \mathrm{k}$ images.

As in [3], we employ the modified MobileNetV1 architecture which has a FC layer with 2 output classes instead of 1000 . The complexity of the network is tuned by varying the network width multiplier[8] $m \in\{0.125,0.25,0.375,0.5\}$. Similar to our ImageNet experiments, we quantize all layers to 8b fixed-point and vary the precision of the PW layers using 8b-to-2b fixed-point and DBQ-1T and DBQ-2T.

As shown in Fig. 6a, for over parameterized models, e.g., $m=0.5$, we find DBQ-1T (red square) shows a massive reduction in $\mathcal{C}_{S}(\sim 69 \%)$ at isoaccuracy compared to the fixed-point models (red circle) (Fig. 6a). In contrast, for lightweight models, e.g., $m=0.125$, DBQ-1T (blue square) achieves an impressive $45 \%$ reduction in $\mathcal{C}_{S}$ but at the expense of a $3 \%$ loss in test accuracy 


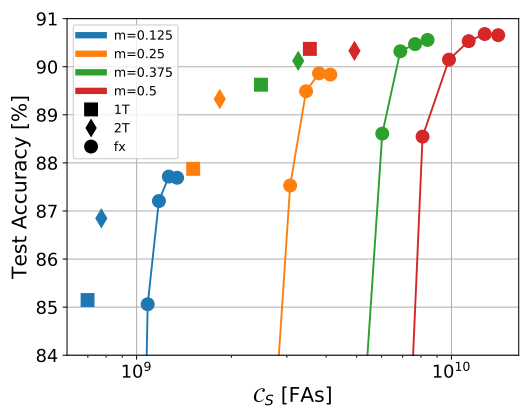

(a)

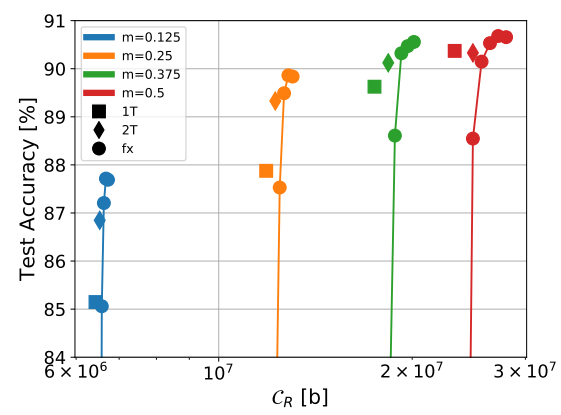

(b)

Fig. 6. The test accuracy of MobileNetV1 on the Visual Wake Words dataset with varying precision assignment and width multiplier $m$ vs. (a) sparsity-aware computational cost, and (b) representational cost. Only the precision of the pointwise layer's weights are changing, whereas all the remaining activations and weights are quantized using $8 \mathrm{~b}$ fixed-point.

as compared to the fixed-point model (blue circle) (Fig. 6a). The DBQ models (diamonds and squares) can be seen to form a pareto-optimal accuracy-vs. $\mathcal{C}_{S}$ trade-off curve in Fig. 6a demonstrating its effectiveness.

Fig. $6 \mathrm{~b}$ shows that the choice of the width multiplier $m$ has a much more significant impact on the representational $\operatorname{cost} \mathcal{C}_{R}$ than varying bit-precision which implies that $\mathcal{C}_{R}$ is dominated by the storage requirements of activations rather than weights. This implies that the choice of the model parameter $m$ is governed by the amount of on-chip storage available on an Edge device. In contrast, the choice of the bit precision of the PW layers is dictated by the latency/energy requirements which upper bounds $\mathcal{C}_{S}$ as seen in Fig. 6a. As a result, when comparing the lightweight $m=0.25 \mathrm{DBQ}-2 \mathrm{~T}$ model (orange diamond) with the over parameterized $m=0.375 \mathrm{DBQ}-1 \mathrm{~T}$ model (green square), we observe that DBQ-2T achieves a reduction in both $\mathcal{C}_{S}(26 \%)$ and $\mathcal{C}_{R}(30 \%)$, at iso-accuracy and iso- $\mathcal{C}_{M}\left(\sim 10^{6} \mathrm{~b}\right)$.

\section{Conclusion}

We presented DBQ, an efficient fully differentiable method for training multiple ternary branch quantizers for deep neural networks and validated its effectiveness for lightweight networks on the CIFAR-10 (ResNet-20), ImageNet (MobileNetV1, MobileNetV2, and ShuffleNetV2) and Visual Wake Words (MobileNetV1) datasets. Our method outperforms the state-of-the-art quantization schemes in both accuracy and complexity metrics.

Acknowledgment: The authors would like to thank Avishek Biswas, Manu Mathew and Arthur Redfern for helpful discussions and support. 


\section{References}

1. Baskin, C., Schwartz, E., Zheltonozhskii, E., Liss, N., Giryes, R., Bronstein, A.M., Mendelson, A.: UNIQ: Uniform noise injection for non-uniform quantization of neural networks. arXiv preprint arXiv:1804.10969 (2018) 2, 3, 5, 12

2. Bengio, Y., Léonard, N., Courville, A.: Estimating or propagating gradients through stochastic neurons for conditional computation. arXiv preprint arXiv:1308.3432 (2013) 3, 4

3. Chowdhery, A., Warden, P., Shlens, J., Howard, A., Rhodes, R.: Visual wake words dataset. arXiv preprint arXiv:1906.05721 (2019) 9, 13

4. Dbouk, H., Geng, H., Vineyard, C.M., Shanbhag, N.R.: Low-complexity fixed-point convolutional neural networks for automatic target recognition. In: ICASSP 20202020 IEEE International Conference on Acoustics, Speech and Signal Processing (ICASSP). pp. 1598-1602. IEEE (2020) 8

5. Han, S., Pool, J., Tran, J., Dally, W.: Learning both weights and connections for efficient neural network. In: Advances in neural information processing systems. pp. 1135-1143 (2015) 2

6. He, K., Zhang, X., Ren, S., Sun, J.: Deep residual learning for image recognition. In: Proceedings of the IEEE conference on computer vision and pattern recognition. pp. $770-778$ (2016) 1, 4, 9, 10

7. Hou, L., Kwok, J.T.: Loss-aware weight quantization of deep networks. arXiv preprint arXiv:1802.08635 (2018) 3

8. Howard, A.G., Zhu, M., Chen, B., Kalenichenko, D., Wang, W., Weyand, T., Andreetto, M., Adam, H.: Mobilenets: Efficient convolutional neural networks for mobile vision applications. arXiv preprint arXiv:1704.04861 (2017) 2, 3, 9, 11, 13

9. Huang, G., Liu, S., Van der Maaten, L., Weinberger, K.Q.: Condensenet: An efficient densenet using learned group convolutions. In: Proceedings of the IEEE Conference on Computer Vision and Pattern Recognition. pp. 2752-2761 (2018) 2

10. Hubara, I., Courbariaux, M., Soudry, D., El-Yaniv, R., Bengio, Y.: Binarized neural networks. In: Advances in neural information processing systems. pp. 4107-4115 (2016) $2,3,4$

11. Iandola, F.N., Han, S., Moskewicz, M.W., Ashraf, K., Dally, W.J., Keutzer, K.: Squeezenet: Alexnet-level accuracy with 50x fewer parameters andi 0.5 mb model size. arXiv preprint arXiv:1602.07360 (2016) 2

12. Ioffe, S., Szegedy, C.: Batch normalization: Accelerating deep network training by reducing internal covariate shift. arXiv preprint arXiv:1502.03167 (2015) 8

13. Jacob, B., Kligys, S., Chen, B., Zhu, M., Tang, M., Howard, A., Adam, H., Kalenichenko, D.: Quantization and training of neural networks for efficient integerarithmetic-only inference. In: Proceedings of the IEEE Conference on Computer Vision and Pattern Recognition. pp. 2704-2713 (2018) 2, 3, 5, 8, 12, 13

14. Krizhevsky, A., Hinton, G., et al.: Learning multiple layers of features from tiny images. Tech. rep., Citeseer (2009) 9

15. Krizhevsky, A., Sutskever, I., Hinton, G.E.: Imagenet classification with deep convolutional neural networks. In: Advances in neural information processing systems. pp. 1097-1105 (2012) 1, 4

16. Li, F., Zhang, B., Liu, B.: Ternary weight networks. arXiv preprint arXiv:1605.04711 (2016) 2, 3, 4

17. Lin, T.Y., Maire, M., Belongie, S., Hays, J., Perona, P., Ramanan, D., Dollár, P., Zitnick, C.L.: Microsoft coco: Common objects in context. In: European conference on computer vision. pp. 740-755. Springer (2014) 13 
18. Lin, X., Zhao, C., Pan, W.: Towards accurate binary convolutional neural network. In: Advances in Neural Information Processing Systems. pp. 345-353 (2017) 3, 4, 7

19. Louizos, C., Reisser, M., Blankevoort, T., Gavves, E., Welling, M.: Relaxed quantization for discretized neural networks. arXiv preprint arXiv:1810.01875 (2018) 2, $3,5,12$

20. Ma, N., Zhang, X., Zheng, H.T., Sun, J.: Shufflenet v2: Practical guidelines for efficient cnn architecture design. In: Proceedings of the European Conference on Computer Vision (ECCV). pp. 116-131 (2018) 3, 9, 11

21. Paszke, A., Gross, S., Chintala, S., Chanan, G., Yang, E., DeVito, Z., Lin, Z., Desmaison, A., Antiga, L., Lerer, A.: Automatic differentiation in PyTorch. In: NIPS Autodiff Workshop (2017) 7

22. Rastegari, M., Ordonez, V., Redmon, J., Farhadi, A.: Xnor-net: Imagenet classification using binary convolutional neural networks. In: European Conference on Computer Vision. pp. 525-542. Springer (2016) 2, 3, 4

23. Russakovsky, O., Deng, J., Su, H., Krause, J., Satheesh, S., Ma, S., Huang, Z., Karpathy, A., Khosla, A., Bernstein, M., et al.: Imagenet large scale visual recognition challenge. International journal of computer vision 115(3), 211-252 (2015) 9

24. Sakr, C., Choi, J., Wang, Z., Gopalakrishnan, K., Shanbhag, N.: True gradientbased training of deep binary activated neural networks via continuous binarization. In: 2018 IEEE International Conference on Acoustics, Speech and Signal Processing (ICASSP). pp. 2346-2350. IEEE (2018) 3

25. Sakr, C., Kim, Y., Shanbhag, N.: Analytical guarantees on numerical precision of deep neural networks. In: Proceedings of the 34th International Conference on Machine Learning-Volume 70. pp. 3007-3016. JMLR. org (2017) 3, 9

26. Sakr, C., Shanbhag, N.: Per-tensor fixed-point quantization of the backpropagation algorithm. In: International Conference on Learning Representations (2019) 9

27. Sandler, M., Howard, A., Zhu, M., Zhmoginov, A., Chen, L.C.: Mobilenetv2: Inverted residuals and linear bottlenecks. In: Proceedings of the IEEE Conference on Computer Vision and Pattern Recognition. pp. 4510-4520 (2018) 2, 3, 9, 11

28. Sheng, T., Feng, C., Zhuo, S., Zhang, X., Shen, L., Aleksic, M.: A quantizationfriendly separable convolution for mobilenets. In: 2018 1st Workshop on Energy Efficient Machine Learning and Cognitive Computing for Embedded Applications (EMC2). pp. 14-18. IEEE (2018) 2, 5, 8, 12

29. Uhlich, S., Mauch, L., Cardinaux, F., Yoshiyama, K., Garcia, J.A., Tiedemann, S., Kemp, T., Nakamura, A.: Mixed precision DNNs: All you need is a good parametrization. In: International Conference on Learning Representations (2020), https://openreview.net/forum?id=Hyx0slrFvH 5, 13

30. Wang, K., Liu, Z., Lin, Y., Lin, J., Han, S.: Haq: Hardware-aware automated quantization with mixed precision. In: Proceedings of the IEEE Conference on Computer Vision and Pattern Recognition. pp. 8612-8620 (2019) 2, 3, 5, 12

31. Xie, S., Zheng, H., Liu, C., Lin, L.: SNAS: stochastic neural architecture search. In: International Conference on Learning Representations (2019), https: //openreview .net/forum?id=rylqooRqK7 6

32. Yang, J., Shen, X., Xing, J., Tian, X., Li, H., Deng, B., Huang, J., Hua, X.s.: Quantization networks. In: Proceedings of the IEEE Conference on Computer Vision and Pattern Recognition. pp. 7308-7316 (2019) 2, 3, 6 
33. Zhang, D., Yang, J., Ye, D., Hua, G.: Lq-nets: Learned quantization for highly accurate and compact deep neural networks. In: Proceedings of the European Conference on Computer Vision (ECCV). pp. 365-382 (2018) 3, 4, 7, 10

34. Zhang, X., Zhou, X., Lin, M., Sun, J.: Shufflenet: An extremely efficient convolutional neural network for mobile devices. In: Proceedings of the IEEE Conference on Computer Vision and Pattern Recognition. pp. 6848-6856 (2018) 2

35. Zhou, A., Yao, A., Wang, K., Chen, Y.: Explicit loss-error-aware quantization for low-bit deep neural networks. In: Proceedings of the IEEE Conference on Computer Vision and Pattern Recognition. pp. 9426-9435 (2018) 3

36. Zhu, C., Han, S., Mao, H., Dally, W.J.: Trained ternary quantization. arXiv preprint arXiv:1612.01064 (2016) 2,3, 4 
Supplementary Material for "DBQ: A Differentiable Branch Quantizer for Lightweight Deep Neural Networks" 


\section{Table of Contents}

Supplementary Material for "DBQ: A Differentiable Branch Quantizer for Lightweight Deep Neural Networks " ...................... 1

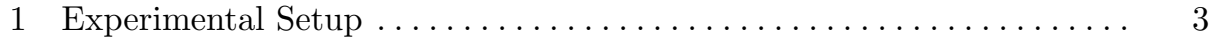

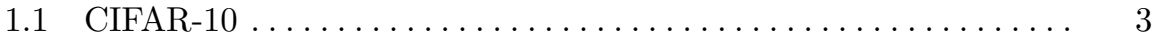

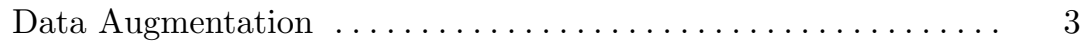

Training Hyperparameters ....................... 3

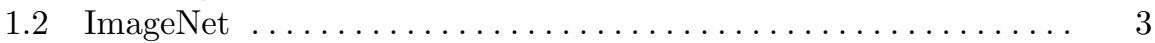

Data Augmentation .............................. 3

Training Hyperparameters . . . . . . . . . . . . . . . . . . 3

1.3 Visual Wake Words . . . . . . . . . . . . . . . . . . . . 4

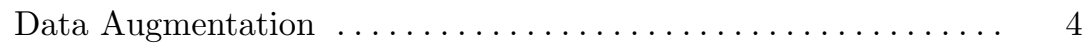

Training Hyperparameters ....................... 4

2 Gradient Derivations . ............................. 4

2.1 Notation ...................................... 5

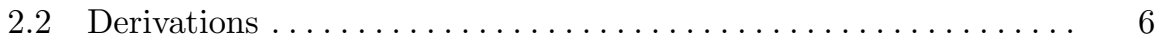

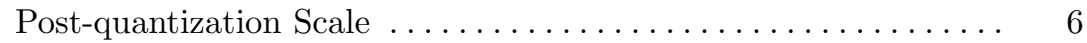

Ternary Branch Scales ........................... 6

Quantizer Thresholds ........................... 6

Pre-quantization Scale ............................ 6

Full Precision Weights .......................... 7

3 MobileNetV2 on ImageNet Comparisons . ................. 7

4 DBQ Branch Sparsity ............................. 7 


\section{Experimental Setup}

In this section, we describe the experimental setup used for generating all our results.

\subsection{CIFAR-10}

Data Augmentation The CIFAR-10 dataset consits of $32 \times 32$ RGB images. For generating the training samples, we adopt the standard data augmentation used in [3] where each image is: 1) zero-padded with 4 pixels on each side; 2) horizontally flipped with probability 0.5 ; and 3 ) randomly cropped using a $32 \times 32$ window. During testing, we use the $32 \times 32$ images as is from the testing set. We also normalize the images, for both training and testing, using a per-channel mean and standard deviation calculated across the training set.

Training Hyperparameters For training the full precision (FP) ResNet-20 baseline on CIFAR-10, we use SGD with momentum $\beta=0.9$, batch size of 100 , and weight decay of $\lambda=10^{-4}$. The FP model is trained for a total of $E_{\mathrm{T}}=200$ epochs, with an initial learning rate $\eta_{0}=0.1$ and a cosine update rule [4]:

$$
\eta_{e}=\frac{\eta_{0}}{2}\left(1+\cos \left(\frac{e}{E_{\mathrm{T}}} \pi\right)\right)
$$

During the fine-tuning process, i.e. training the model with weights initialized from the FP baseline, we train using the same setup as before, but for a fewer number of epochs $E_{\mathrm{T}}=50$ and a smaller initial learning rate $\eta_{0}=0.01$. The DBQ models trained use a linear temperature increment schedule:

$$
T_{e}=T_{\mathrm{init}}+e \cdot T_{\mathrm{inc}}
$$

with an initial temperature $T_{\text {init }}=5$ and increments $T_{\text {inc }}=2.5$.

\subsection{ImageNet}

Data Augmentation For our ImageNet experiments, we follow the standard data augmentation used in [2], where during training, images are: 1) resized; 2) horizontally flipped; and 3) randomly cropped to $224 \times 224$. During testing, all images are resized to $256 \times 256$ and then cropped to $224 \times 224$. We also normalize the input images on a per-channel basis.

Training Hyperparameters For training the full precision MobileNetV1 baseline on ImageNet, we use a similar setup as our CIFAR-10 experiments, with a slightly different learning rate schedule. Similar to [1], the first $E_{\mathrm{W}}$ epochs are used for learning rate "warm-up":

$$
\eta_{e}=\frac{(e+1) \eta_{0}}{E_{W}}
$$


after which the remaining epochs utilize a cosine learning rate as described in (1). The hyperparameters used for both FP and quantization fine-tuning are specified in Table 1.

The full precision MobileNetV2 and ShuffleNetV2 baselines on ImageNet are pre-trained models obtained from PyTorch [5]. Their 2T quantized counterparts, MobileNetV2-2T and ShuffleNetV2-2T, are fine-tuned using the training hyperparameters described in Table 2.

\begin{tabular}{|c||c|c|c|c|c|c||c|c|}
\hline & Batch Size & $\beta$ & $\lambda$ & $\eta_{0}$ & $E_{\mathrm{W}}$ & $E_{\mathrm{T}}$ & $T_{\text {init }}$ & $T_{\text {inc }}$ \\
\hline \hline FP & 512 & 0.9 & $4 \times 10^{-5}$ & 0.1 & 5 & 150 & NA & NA \\
\hline Quant. & 512 & 0.9 & $4 \times 10^{-5}$ & 0.001 & 0 & 50 & 50 & 20 \\
\hline
\end{tabular}

Table 1. Training hyperparameters used for MobileNetV1 experiments on the ImageNet dataset.

\begin{tabular}{|l||c|c|c|c|c|c||c|c|}
\hline & Batch Size & $\beta$ & $\lambda$ & $\eta_{0}$ & $E_{\mathrm{W}}$ & $E_{\mathrm{T}}$ & $T_{\text {init }}$ & $T_{\text {inc }}$ \\
\hline MobileNetV2-2T & 256 & 0.9 & $4 \times 10^{-5}$ & $5 \times 10^{-4}$ & 0 & 50 & 25 & 10 \\
\hline ShuffleNetV2-2T & 512 & 0.9 & $4 \times 10^{-5}$ & 0.001 & 0 & 30 & 25 & 10 \\
\hline
\end{tabular}

Table 2. Training hyperparameters used for quantized MobileNetV2 and ShuffleNetV2 experiments on the ImageNet dataset.

\subsection{Visual Wake Words}

Data Augmentation For data augmentation during training, we follow the exact setup as our ImageNet experiments with input normalization and random horizontal flips and crops. During testing, images are normalized, resized to $256 \times 256$, and then cropped to $224 \times 224$.

Training Hyperparameters The training setup used is identical to our ImageNet experiments as well, and Table 3 specifies the values of the hyperparameters used for both full precision and quantization training.

\section{Gradient Derivations}

In this section, we provide derivations for the gradient expressions of the loss function $\mathcal{L}$ with respect to the full precision weights $\mathbf{w} \in \mathbb{R}^{D}$ and the quantizer 


\begin{tabular}{|c||c|c|c|c|c|c|c|c|}
\hline & Batch Size & $\beta$ & $\lambda$ & $\eta_{0}$ & $E_{\mathrm{W}}$ & $E_{\mathrm{T}}$ & $T_{\text {init }}$ & $T_{\text {inc }}$ \\
\hline \hline FP & 512 & 0.9 & $4 \times 10^{-5}$ & 0.1 & 5 & 200 & NA & NA \\
\hline Quant. & 512 & 0.9 & $4 \times 10^{-5}$ & 0.01 & 0 & 50 & 20 & 5 \\
\hline
\end{tabular}

Table 3. Training hyperparameters used for experiments on the Visual Wake Words dataset.

parameters $\mathcal{P}_{Q}=\left\{\alpha_{1}, \ldots, \alpha_{B}, \gamma_{1}, \gamma_{2}, t_{1}, \ldots, t_{N-1}\right\}$. Recall that during training, the quantizer expression is:

$$
\mathbf{z}=Q_{T}(\mathbf{w})=\gamma_{2}\left[\sum_{i=1}^{N-1}\left[\hat{f}_{T}\left(\gamma_{1} \mathbf{w}-t_{i}\right) \sum_{j=1}^{B} b_{i, j} \alpha_{j}\right]-\sum_{j=1}^{B} \alpha_{j}\right]
$$

where $\hat{f}_{T}$ is the smooth approximation using the Sigmoid function:

$$
\hat{f}_{T}(u)=\frac{1}{1+\exp (-T u)}
$$

whose derivative can be easily written as:

$$
\frac{\partial \hat{f}_{T}(u)}{\partial u}=T \hat{f}_{T}(u)\left[1-\hat{f}_{T}(u)\right]
$$

\subsection{Notation}

The derivations of these gradients involves computing derivatives with vectors. Thus, in this section we establish the appropriate notation. The derivative of a scalar $y$ with respect to a $D$-dimensional vector $\mathbf{x}$ is:

$$
\frac{\partial y}{\partial \mathbf{x}}=\left[\frac{\partial y}{\partial x_{1}} \frac{\partial y}{\partial x_{2}} \ldots \frac{\partial y}{\partial x_{D}}\right]
$$

whereas the derivative of a vector $\mathbf{y}$ with respect to a scalar $x$ is:

$$
\frac{\partial \mathbf{y}}{\partial x}=\left[\begin{array}{c}
\frac{\partial y_{1}}{\partial x} \\
\frac{\partial y_{2}}{\partial x} \\
\vdots \\
\frac{\partial y_{D}}{\partial x}
\end{array}\right]
$$

The derivative of a scalar $y$ with respect to another scalar $x$, assuming $y=g(\mathbf{z})$ and $\mathbf{z}=f(x)$, can therefore be computed using the chain rule:

$$
\frac{\partial y}{\partial x}=\frac{\partial y}{\partial \mathbf{z}} \cdot \frac{\partial \mathbf{z}}{\partial x}=\left[\begin{array}{lll}
\frac{\partial y}{\partial z_{1}} & \frac{\partial y}{\partial z_{2}} \ldots \frac{\partial y}{\partial z_{D}}
\end{array}\right]\left[\begin{array}{c}
\frac{\partial z_{1}}{\partial x} \\
\frac{\partial z_{2}}{\partial x} \\
\vdots \\
\frac{\partial z_{D}}{\partial x}
\end{array}\right]=\sum_{k=1}^{D} \frac{\partial y}{\partial z_{k}} \cdot \frac{\partial z_{k}}{\partial x}
$$




\subsection{Derivations}

Post-quantization Scale We notice that:

$$
\frac{\partial z_{k}}{\partial \gamma_{2}}=\frac{z_{k}}{\gamma_{2}}
$$

which can be plugged in to get the gradient using the chain rule:

$$
\frac{\partial \mathcal{L}}{\partial \gamma_{2}}=\frac{\partial \mathcal{L}}{\partial \mathbf{z}} \cdot \frac{\partial \mathbf{z}}{\partial \gamma_{2}}=\sum_{k=1}^{D} \frac{\partial \mathcal{L}}{\partial z_{k}} \cdot \frac{\partial z_{k}}{\partial \gamma_{2}}=\frac{1}{\gamma_{2}} \sum_{k=1}^{D} \frac{\partial \mathcal{L}}{\partial z_{k}} z_{k}
$$

Ternary Branch Scales We first compute $\forall j \in[B]$ :

$$
\frac{\partial z_{k}}{\partial \alpha_{j}}=\gamma_{2}\left[\sum_{i=1}^{N-1}\left[\hat{f}_{T}\left(\gamma_{1} w_{k}-t_{i}\right) b_{i, j}\right]-1\right]=\gamma_{2}\left[\sum_{i=1}^{N-1}\left[g_{k, i} b_{i, j}\right]-1\right]
$$

where $g_{k, i}=\hat{f}_{T}\left(\gamma_{1} w_{k}-t_{i}\right)$ for brevity. Therefore, using the chain rule we obtain:

$$
\frac{\partial \mathcal{L}}{\partial \alpha_{j}}=\frac{\partial \mathcal{L}}{\partial \mathbf{z}} \cdot \frac{\partial \mathbf{z}}{\partial \alpha_{j}}=\sum_{k=1}^{D} \frac{\partial \mathcal{L}}{\partial z_{k}} \cdot \frac{\partial z_{k}}{\partial \alpha_{j}}=\gamma_{2} \sum_{k=1}^{D} \frac{\partial \mathcal{L}}{\partial z_{k}}\left[\sum_{i=1}^{N-1}\left[b_{i, j} g_{k, i}\right]-1\right]
$$

Quantizer Thresholds We first utilize (6) in order to compute $\forall i \in[N-1]$ :

$$
\begin{aligned}
\frac{\partial z_{k}}{\partial t_{i}} & =\gamma_{2}\left[\frac{\partial \hat{f}_{T}\left(\gamma_{1} w_{k}-t_{i}\right)}{\partial t_{i}} \sum_{j=1}^{B} b_{i, j} \alpha_{j}\right] \\
& =-\gamma_{2} T\left[g_{k, i}\left(1-g_{k, i}\right) \sum_{j=1}^{B} b_{i, j} \alpha_{j}\right]=-\gamma_{2} T\left[h_{k, i} \sum_{j=1}^{B} b_{i, j} \alpha_{j}\right]
\end{aligned}
$$

where $h_{k, i}=g_{k, i}\left(1-g_{k, i}\right)$ for brevity. Therefore using the chain rule we obtain:

$$
\frac{\partial \mathcal{L}}{\partial t_{i}}=\frac{\partial \mathcal{L}}{\partial \mathbf{z}} \cdot \frac{\partial \mathbf{z}}{\partial t_{i}}=\sum_{k=1}^{D} \frac{\partial \mathcal{L}}{\partial z_{k}} \cdot \frac{\partial z_{k}}{\partial t_{i}}=-\gamma_{2} T \sum_{k=1}^{D} \frac{\partial \mathcal{L}}{\partial z_{k}}\left[h_{k, i} \sum_{j=1}^{B} b_{i, j} \alpha_{j}\right]
$$

Pre-quantization Scale Similarly, we utilize (6) in order to compute:

$$
\frac{\partial z_{k}}{\partial \gamma_{1}}=\gamma_{2}\left[\sum_{i=1}^{N-1}\left[\frac{\partial \hat{f}_{T}\left(\gamma_{1} w_{k}-t_{i}\right)}{\partial \gamma_{1}} \sum_{j=1}^{B} b_{i, j} \alpha_{j}\right]\right]=\gamma_{2} T w_{k}\left[\sum_{i=1}^{N-1}\left[h_{k, i} \sum_{j=1}^{B} b_{i, j} \alpha_{j}\right]\right]
$$

and therefore applying the chain rule yields:

$$
\frac{\partial \mathcal{L}}{\partial \gamma_{1}}=\frac{\partial \mathcal{L}}{\partial \mathbf{z}} \cdot \frac{\partial \mathbf{z}}{\partial \gamma_{1}}=\sum_{k=1}^{D} \frac{\partial \mathcal{L}}{\partial z_{k}} \cdot \frac{\partial z_{k}}{\partial \gamma_{1}}=\gamma_{2} T \sum_{k=1}^{D} \frac{\partial \mathcal{L}}{\partial z_{k}} w_{k}\left[\sum_{i=1}^{N-1}\left[h_{k, i} \sum_{j=1}^{B} b_{i, j} \alpha_{j}\right]\right]
$$


Full Precision Weights Finally, in order to compute the gradient of $\mathcal{L}$ with respect to the full precision weights $\mathbf{w}=\left[w_{1}, \ldots, w_{D}\right]^{\mathrm{T}}$, we first compute $\forall k \in[D]$ :

$$
\begin{aligned}
\frac{\partial z_{m}}{\partial w_{k}} & =\gamma_{2}\left[\sum_{i=1}^{N-1}\left[\frac{\partial \hat{f}_{T}\left(\gamma_{1} w_{m}-t_{i}\right)}{\partial w_{k}} \sum_{j=1}^{B} b_{i, j} \alpha_{j}\right]\right] \\
& = \begin{cases}\gamma_{1} \gamma_{2} T\left[\sum_{i=1}^{N-1}\left[h_{k, i} \sum_{j=1}^{B} b_{i, j} \alpha_{j}\right]\right], & \text { if } m=k \\
0, & \text { otherwise }\end{cases}
\end{aligned}
$$

and using the chain rule, we obtain:

$$
\frac{\partial \mathcal{L}}{\partial w_{k}}=\frac{\partial \mathcal{L}}{\partial \mathbf{z}} \cdot \frac{\partial \mathbf{z}}{\partial w_{k}}=\sum_{m=1}^{D} \frac{\partial \mathcal{L}}{\partial z_{m}} \cdot \frac{\partial z_{m}}{\partial w_{k}}=\gamma_{1} \gamma_{2} T \frac{\partial \mathcal{L}}{\partial z_{k}} \sum_{i=1}^{N-1}\left[h_{k, i} \sum_{j=1}^{B} b_{i, j} \alpha_{j}\right]
$$

\section{MobileNetV2 on ImageNet Comparisons}

We compare DBQ and [6] on MobileNetV2 in Table 4. [6] has two versions trained models M1 and M2, where M1 is trained with a memory constraint and M2 is not. We find that DBQ-2T is smaller than M2 [6] at iso-accuracy on ImageNet and more accurate than M1 [6] but at a larger storage cost. We are unable to compare the computational complexities since [6] lacks sufficient information, hence we adopt the metrics reported in [6], which are weight storage (analogous to $\mathcal{C}_{M}$ ) and activation storage (analogous to $\mathcal{C}_{R}-\mathcal{C}_{M}$ ).

\begin{tabular}{l|ccc}
\hline Model & Top-1 Acc. [\%] & Weight Storage $[\mathrm{MB}]$ & Activation Storage [MB] \\
\hline M1 [6] (w/ constr.) & 69.74 & 1.55 & 0.57 \\
\hline M2 [6] (w/o constr.) & 70.59 & 3.14 & 1.58 \\
\hline DBQ-2T & $\mathbf{7 0 . 5 4}$ & $\mathbf{2 . 4 3}$ & $\mathbf{1 . 1 5}$ \\
\hline
\end{tabular}

Table 4. The Top-1 accuracy on ImageNet and Storage costs for MobileNetV2 using our method (DBQ-2T) compared to [6].

\section{DBQ Branch Sparsity}

One of the advantages of implementing ternary-based dot products is leveraging weight sparsity, which is reflected in our sparsity-aware computational $\operatorname{cost} \mathcal{C}_{S}$. In this work, we show that for MobileNetV1 on ImageNet with two ternary branch quantization (DBQ-2T-4), the computational cost can be reduced from $2.18 \times$ $10^{10}$ FAs to $1.42 \times 10^{10}(\sim 35 \%$ reduction $)$ by simply skipping the operations involving zero weights. Table 5 reports the average branch level sparsity for 


\begin{tabular}{|c||c|c|c|c|c|}
\cline { 3 - 6 } \multicolumn{2}{c}{} & \multicolumn{3}{c|}{ Average Branch Sparsity [\%] } \\
\hline PW Layer & $C_{\text {in }}$ & $C_{\text {out }}$ & FX8 & DBQ-1T & DBQ-2T \\
\hline \hline 0 & 64 & 32 & 35.55 & 58.69 & 64.82 \\
\hline 1 & 64 & 128 & 10.74 & 41.42 & 51.75 \\
\hline 2 & 128 & 128 & 6.86 & 34.09 & 46.45 \\
\hline 3 & 128 & 256 & 6.73 & 31.83 & 44.96 \\
\hline 4 & 256 & 256 & 4.53 & 29.10 & 43.05 \\
\hline 5 & 256 & 512 & 7.31 & 30.62 & 44.36 \\
\hline 6 & 512 & 512 & 6.41 & 28.50 & 43.40 \\
\hline 7 & 512 & 512 & 6.00 & 26.48 & 42.94 \\
\hline 8 & 512 & 512 & 4.00 & 24.03 & 41.70 \\
\hline 9 & 512 & 512 & 5.57 & 24.89 & 42.56 \\
\hline 10 & 512 & 512 & 5.50 & 23.65 & 42.30 \\
\hline 11 & 512 & 1024 & 7.00 & 23.17 & 42.41 \\
\hline 12 & 1024 & 1024 & 10.69 & 28.25 & 45.77 \\
\hline \hline Network & Average & 7.59 & 26.50 & $\mathbf{4 3 . 7 8}$ \\
\hline
\end{tabular}

Table 5. Branch level sparsity for all the pointwise (PW) layers of MobileNetV1 on ImageNet. $C_{\text {in }}$ and $C_{\text {out }}$ denote the number of input and output channels respectively.

every point wise layer. For the DBQ-2T model, which quantizes PW layers to two ternary branches, we find that on average $43.78 \%$ of all PW weights are zero, which explains the massive $35 \%$ reduction in $\mathcal{C}_{S}$. In contrast, the DBQ-1T model, which quantizes all PW layers to one ternary branch, achieves a $26.5 \%$ average branch sparsity. While DBQ-2T has twice the number of branches compared to DBQ-1T, the per-branch sparsity is actually much higher for the DBQ-2T. In other words, while the number of pointwise parameters increases by $2 \times$ when going from $1 \mathrm{~T}$ to $2 \mathrm{~T}$, due to the high branch sparsity, the number of non-zero parameters increases by $1.53 \times$ only. On the other hand, using $8 \mathrm{~b}$ fixed-point for the PW layers yields very little weight sparsity (7.59\%).

\section{References}

1. Goyal, P., Dollár, P., Girshick, R., Noordhuis, P., Wesolowski, L., Kyrola, A., Tulloch, A., Jia, Y., He, K.: Accurate, large minibatch SGD: Training imagenet in 1 hour. arXiv preprint arXiv:1706.02677 (2017) 3

2. He, K., Zhang, X., Ren, S., Sun, J.: Deep residual learning for image recognition. In: Proceedings of the IEEE conference on computer vision and pattern recognition. pp. $770-778$ (2016) 3

3. Huang, G., Liu, S., Van der Maaten, L., Weinberger, K.Q.: Condensenet: An efficient densenet using learned group convolutions. In: Proceedings of the IEEE Conference on Computer Vision and Pattern Recognition. pp. 2752-2761 (2018) 3

4. Loshchilov, I., Hutter, F.: SGDR: Stochastic gradient descent with warm restarts. arXiv preprint arXiv:1608.03983 (2016) 3 
5. Paszke, A., Gross, S., Chintala, S., Chanan, G., Yang, E., DeVito, Z., Lin, Z., Desmaison, A., Antiga, L., Lerer, A.: Automatic differentiation in PyTorch. In: NIPS Autodiff Workshop (2017) 4

6. Uhlich, S., Mauch, L., Cardinaux, F., Yoshiyama, K., Garcia, J.A., Tiedemann, S., Kemp, T., Nakamura, A.: Mixed precision DNNs: All you need is a good parametrization. In: International Conference on Learning Representations (2020), https://openreview.net/forum?id=Hyx0slrFvH 7 